\title{
Free expansion of attractive and repulsive Bose-Einstein condensed vortex states
}

\author{
Sadhan K. Adhikari \\ Instituto de Física Teórica, Universidade Estadual Paulista, 01.405-900 São Paulo, São Paulo, Brazil
}

(Received 11 September 2001; published 26 February 2002)

\begin{abstract}
Free expansion of attractive and repulsive Bose-Einstein condensed vortex states formed in an axially symmetric trap is investigated using the numerical solution of the time-dependent Gross-Pitaevskii equation. In a repulsive condensate the vortex-core radius is much smaller than the radial root-mean-square (rms) radius, which makes the experimental observation of the vortex core difficult. The opposite is found to be true in an attractive condensate that makes it a better candidate for experimental observation. Also, in all cases the ratio of vortex-core radius to radial rms radius increases as the angular momentum of the vortex increases. This makes the vortex states with higher angular momenta more suitable for experimental confirmation.
\end{abstract}

DOI: 10.1103/PhysRevA.65.033616

PACS number(s): 03.75.Fi

\section{INTRODUCTION}

Since the successful detection [1] of Bose-Einstein condensates (BEC) in dilute bosonic atoms employing magnetic trap at ultralow temperature, one problem of extreme interest is the formation of vortex states in an axially symmetric trap. The recent experimental detection [2] of vortex states in the condensate has intensified this endeavor. There have been many theoretical studies on different aspects of BEC [3-5] and specially, on the vortex states in axially symmetric traps [6-14].

The study of the free expansion of a vortex state is of utmost importance as such an expansion can aid in its experimental confirmation [7]. The specific behavior of the time evolution of vortex core and the radial root-meansquare (rms) radius of the condensate after the removal of the trap could be fundamental in the identification of vortex states. However, a previous theoretical study on the free expansion of vortex states was limited to condensates with repulsive interatomic interaction [7]. In this paper we extend this effort to condensates with attractive interatomic interaction and compare critically the expansion dynamics with that for repulsive interaction. This is of extreme experimental relevance as by changing the external electromagnetic field it is now possible to change the effective interatomic interaction in a controlled way from repulsive to attractive exploiting a Feshbach resonance [15].

We base our study on the numerical solution $[4,6,7,14]$ of the nonlinear time-dependent mean-field Gross-Pitaevskii (GP) equation [16] with an axially symmetric trap, which should provide a faithful description of the formation and evolution of vortex states $[7,14]$.

The study of superfluid properties of BEC is of great interest to both theoreticians and experimentalists [8,9]. Quantized vortex state in BEC is intimately connected to the existence of superfluidity. Such vortices are expected in superfluid He II. However, due to strong interaction between the helium atoms there is no reliable mean-field description and a controlled theoretical study of the dynamics is not possible.

On the other hand, a weakly interacting trapped BEC is well described by the mean-field GP equation that is known to admit vortex solutions for a trap with cylindrical symme- try [6], which can be studied numerically. In contrast to superfluid He II, the condensed vortex state of trapped BEC is an extremely dilute quantum fluid and permits a mean-field description. Moreover, vortex states in BEC have been observed experimentally [2]. Many different techniques for creating vortex states in BEC have been suggested [9], e.g., stirring the BEC with an external laser [8], forming spontaneously in evaporative cooling [11], using a "phase imprinting" method [12], and rotating of axially symmetric trap [13].

It is possible to have dynamically stable vortex BEC states with low quanta of rotational excitation or angular momentum $L$ per particle for both attractive $[6,13]$ and repulsive [14] atomic interactions. However, in the attractive case such states exist for the number of atoms below a critical number that increases with $L$. In the absence of vortex $(L=0)$, the stable condensate in a cylindrical trap has a cylindrical shape. Such a BEC has the largest density on the axis of the trap. In the presence of vortex motion the region of largest density of the BEC with nonzero $L$ is pushed away from the central axial region and the atoms have more space to stabilize. The vortex state of the condensate in a cylindrical trap has the shape of a hollow cylinder with zero density on the axis of symmetry.

For vortex states with low angular quanta $L$ and large number of atoms, the radius of the hollow part is much smaller than the radius of the condensate in the radial direction, which makes the experimental confirmation of vortex states difficult. It has been argued and established in repulsive condensates with $L=1$ that after a free expansion, the hollow core of the vortex may expand faster than the radius of the condensate and thus make the internal hollow region detectable experimentally confirming a vortex state [7].

In our study of free expansion of vortex states we find that the attractive condensates allow vortex states with a different nature from those in repulsive condensates. For a fixed $L$, the ratio of vortex core radius to radial rms radius of an attractive condensate is always larger than the same ratio for a repulsive condensate, which may make attractive condensates more appealing for generating, detecting, and studying vortex states. We also find that for a fixed $L$, upon free expansion the above ratio increases for repulsive condensates, whereas it decreases for attractive condensates remaining, 
however, always larger than the corresponding ratio for repulsive condensates. In all cases the ratio increases with $L$.

The present study is performed with the direct numerical solution of the time-dependent GP equation with an axially symmetric trap. In the time-evolution of the GP equation the radial and axial variables are dealt with in two independent steps. In each step the GP equation is solved by discretization with the Crank-Nicholson rule complemented by the known boundary conditions [14,17]. We find that this timedependent approach leads to good convergence.

In Sec. II we describe briefly the time-dependent GP equation with vortex states and a numerical method for its solution. In Sec. III we report the numerical results of the present investigation about the free expansion of vortex states and finally, in Sec. IV we give a summary of our investigation.

\section{NONLINEAR GROSS-PITAEVSKII EQUATION}

The details of the calculational procedure based on the GP equation has been elaborated elsewhere [14] and we present a summary of our account here. At zero temperature, the time-dependent Bose-Einstein condensate wave function $\Psi(\mathbf{r} ; \tau)$ at position $\mathbf{r}$ and time $\tau$ may be described by the following mean-field nonlinear GP equation $[5,16]$ :

$$
\left[-\frac{\hbar^{2}}{2 m} \nabla^{2}+V(\mathbf{r})+g N|\Psi(\mathbf{r} ; \tau)|^{2}-i \hbar \frac{\partial}{\partial \tau}\right] \Psi(\mathbf{r} ; \tau)=0 .
$$

Here $m$ is the mass and $N$ the number of atoms in the condensate, $g=4 \pi \hbar^{2} a / m$ the strength of interatomic interaction, with $a$ the atomic scattering length. The trap potential with cylindrical symmetry may be written as $V(\mathbf{r})$ $=\frac{1}{2} m \omega^{2}\left(r^{2}+\lambda^{2} z^{2}\right)$ where $\omega$ is the angular frequency in the radial direction $r$ and $\lambda \omega$ that in the axial direction $z$. We are using the cylindrical coordinate system $\mathbf{r} \equiv(r, \theta, z)$ with $\theta$ the azimuthal angle. The normalization condition of the wave function is $\int d \mathbf{r}|\Psi(\mathbf{r} ; \tau)|^{2}=1$.

The GP equation (2.1) can accommodate quantized vortex states with rotational motion of the condensate around the $z$ axis. In such a vortex the atoms flow with tangential velocity $L \hbar /(m r)$ such that each atom has quantized angular momentum $L \hbar$ along $z$ axis. This corresponds to the angular dependence

$$
\Psi(\mathbf{r} ; \tau)=\psi(r, z ; \tau) \exp (i L \theta)
$$

of the wave function.

Substituting Eq. (2.2) into Eq. (2.1), and transforming to dimensionless variables defined by $x=\sqrt{2} r / l, y=\sqrt{2} z / l, t$ $=\tau \omega, l \equiv \sqrt{\hbar /(m \omega)}$, and

$$
\phi(x, y ; t) \equiv \frac{\varphi(x, y ; t)}{x}=\sqrt{\frac{l^{3}}{\sqrt{8}}} \psi(r, z ; \tau),
$$

we get

$$
\begin{gathered}
{\left[-\frac{\partial^{2}}{\partial x^{2}}+\frac{1}{x} \frac{\partial}{\partial x}-\frac{\partial^{2}}{\partial y^{2}}+\frac{L^{2}}{x^{2}}+\frac{1}{4}\left(x^{2}+\lambda^{2} y^{2}-\frac{4}{x^{2}}\right)\right.} \\
\left.+8 \sqrt{2} \pi n\left|\frac{\varphi(x, y ; t)}{x}\right|^{2}-i \frac{\partial}{\partial t}\right] \varphi(x, y ; t)=0,
\end{gathered}
$$

where $n=N a / l$. The normalization condition of the wave function becomes

$$
2 \pi \int_{0}^{\infty} d x \int_{-\infty}^{\infty} d y|\varphi(x, y ; t)|^{2} x^{-1}=1 .
$$

For a stationary solution the time dependence of the wave function is given by $\varphi(x, y ; t)=\exp (-i \mu t) \varphi(x, y)$, where $\mu$ is the chemical potential of the condensate in units of $\hbar \omega$. If we use this form of the wave function in Eq. (2.4), we obtain the following stationary nonlinear time-independent GP equation [16]:

$$
\begin{gathered}
{\left[-\frac{\partial^{2}}{\partial x^{2}}+\frac{1}{x} \frac{\partial}{\partial x}-\frac{\partial^{2}}{\partial y^{2}}+\frac{L^{2}}{x^{2}}+\frac{1}{4}\left(x^{2}+\lambda^{2} y^{2}-\frac{4}{x^{2}}\right)\right.} \\
\left.+8 \sqrt{2} \pi n\left|\frac{\varphi(x, y)}{x}\right|^{2}-\mu\right] \varphi(x, y)=0 .
\end{gathered}
$$

The rms radii of the condensate in the radial and axial directions are defined, respectively, by

$$
\left(x_{\mathrm{rms}}\right)^{2}=2 \pi \int_{0}^{\infty} d x \int_{-\infty}^{\infty} d y x|\varphi(x, y ; t)|^{2},
$$

and

$$
\left(y_{\mathrm{rms}}\right)^{2}=2 \pi \int_{0}^{\infty} d x \int_{-\infty}^{\infty} d y x^{-1} y^{2}|\varphi(x, y ; t)|^{2}
$$

We solve the GP equation (2.4) using a time-iteration method elaborated in Refs. [14,17]. The full GP Hamiltonian is conveniently broken into two parts $H_{x}$ and $H_{y}$-the first containing the $x$-dependent terms and the second containing the $y$-dependent terms with the nonlinear interaction term divided equally into two parts. The GP equations along the $x$ and $y$ directions are defined on a two-dimensional set of grid points $N_{x} \times N_{y}$ using the Crank-Nicholson discretization method. The resultant tridiagonal equations along $x$ and $y$ directions are solved alternately by the Gaussian elimination method along the $x$ and $y$ directions [17]. Effectively, each time iteration of the GP equation is broken up into two parts-first using $H_{x}$ and then $H_{y}$. For a small time step $\Delta$, the error involved in this breakup procedure along $x$ and $y$ directions is quadratic in $\Delta$ and hence can be neglected. This scheme is repeated for about 500 time iterations to yield the final solution.

The initial solution for the iterative scheme is taken to be the analytic solution of the corresponding free harmonic oscillator setting the nonlinear term to zero in the GP equation (2.6). At each iteration the strength of the nonlinear term is increased by a small amount so that after the final iteration 
the full strength is attained and the required solution of the GP equation is obtained. The free expansion of the vortex states is studied essentially using the above iterative scheme by setting the trapping potential to zero in the GP equation after the final solution for the vortex state is obtained.

The typical value of the space step used for discretization along $x$ and $y$ directions is 0.02 and that for time step $\Delta$ is 0.05 . For small nonlinearity the largest values of $x$ and $y$ are $x_{\max }=8,|y|_{\max }=8$. However, for stronger nonlinearity, larger values of $x_{\max }$ and $|y|_{\max }$ (up to 20) are employed.

\section{NUMERICAL RESULT}

We present results for the free expansion of vortex states by using the numerical solution of the time-dependent GP equation for attractive and repulsive interatomic interactions for different $L$. In a recent study of repulsive vortex states with $L=1$, Dalfovo and Modugno [7] pointed out that the small vortex-core radius of these condensates make the vortex states hard to detect experimentally. They show that after a free expansion, the vortex-core radius increases faster than the radial rms radius, which makes the experimental observation of the vortex core easier. Here we complement the above study by including the attractive condensates as well as vortex states with $L>1$. The interesting features of the results that we emphasize here are quite independent of the value of axial to radial trap frequency $\lambda$, and in this study we present results for $\lambda=\sqrt{8}$ only.

In Figs. 1(a) and 1(b) we plot the profile of the section of the wave function $|\phi(x, 0 ; t)| \equiv|\varphi(x, 0 ; t)| / x$ upon free expansion as a function of $x$ at different times $0<t<3$ for $n$ $=-1.5$ (attractive case) and $n=12$ (repulsive case), respectively; the trap being removed at time $t=0$. In the attractive case, the minimum value of $n$ allowed for $L=1$ is -1.63 [14]. A vortex-core radius $x_{c}$ is conveniently defined to be the smaller value of $x$ where the central core density $|\phi(x, 0 ; t)|^{2}$ attains $e^{-1}$ times the peak value in Figs. 1(a) and 1(b). After free expansion, both the core radius $x_{c}$ and radial rms radius of the condensate increase. The relative increase of these two radii is crucial in the experimental observation of the vortex states. For this a careful study of the evolution of the different radii is important. In Fig. 2 we present the time evolution of $x_{\mathrm{rms}}$ and $y_{\mathrm{rms}}$, where we plot $x_{\mathrm{rms}}(t) / x_{\mathrm{rms}}(0)$ and $y_{\mathrm{rms}}(t) / y_{\mathrm{rms}}(0)$ vs $t$ for $L=1$ and $n$ $=-1.5,0,12$. Both ratios increase with time in all cases. In the attractive case the radius in the radial direction increases faster, the opposite being true in the repulsive case. The result for $n=0$ always stays between the attractive and repulsive cases.

Next we study the evolution of the ratio $x_{c} / x_{\text {rms }}$, always less than unity, with time $t$. The larger this ratio, the larger is the vortex-core radius compared to the radial rms radius and the easier could be the experimental detection of the vortex state. In Fig. 3 we plot $x_{c} / x_{\text {rms }}$ vs time $t$ for $L=1$ and 4 for several $n$ including the attractive (negative $n$ ), repulsive (positive $n$ ) and noninteracting ( $n=0)$ cases. For fixed $n$ and $t$, this ratio increases with $L$; and for fixed $L$ and $t$, the ratio increases as $n$ decreases from positive to negative values (passes from repulsive to attractive). This means that for at-
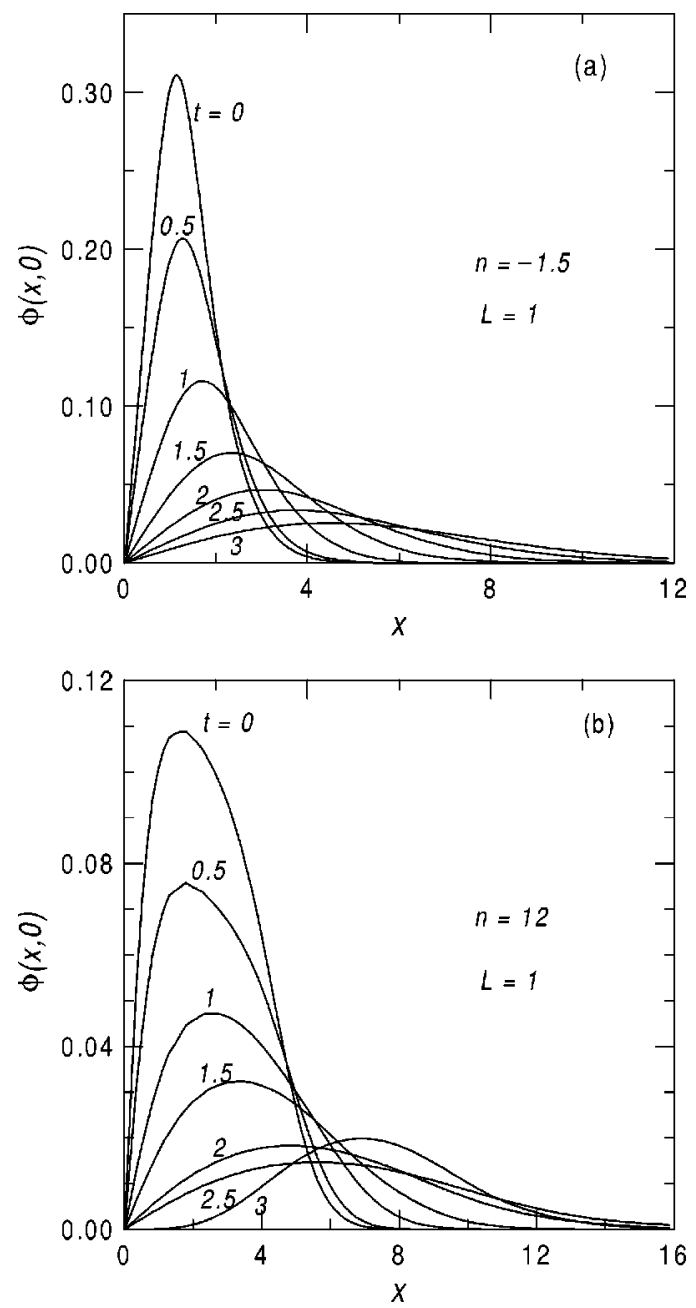

FIG. 1. Wave function $\phi(x, y=0)$ of a stable vortex state with $L=1$ vs $x$ for (a) $n=-1.5$, and (b) $n=12$.

tractive interaction the vortex state has a larger vortex-core radius compared to the radial rms radius and hence could be easily detected. From Fig. 3 we find that at $t=0$ the numerical value of this ratio for attractive condensate could be twice

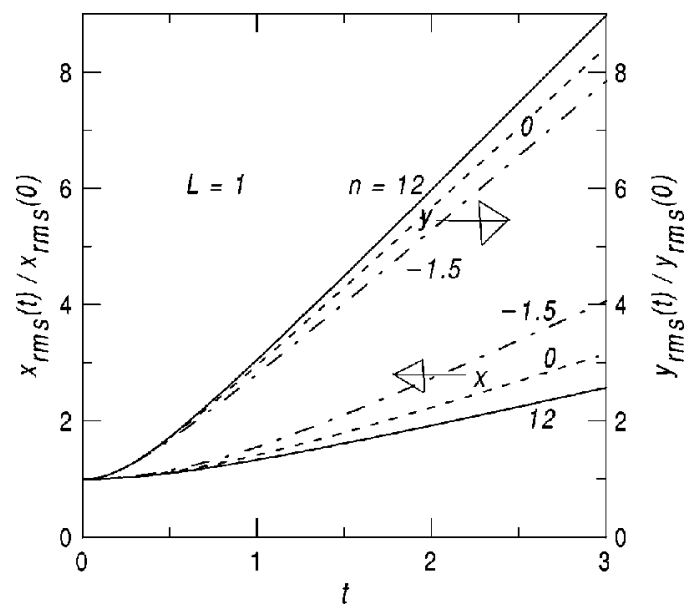

FIG. 2. The ratios $x_{\mathrm{rms}}(t) / x_{\mathrm{rms}}(0)$ and $y_{\mathrm{rms}}(t) / y_{\mathrm{rms}}(0)$ vs time $t$ of expanding vortex states with $L=1$ and $n=-1.5,0,12$. 


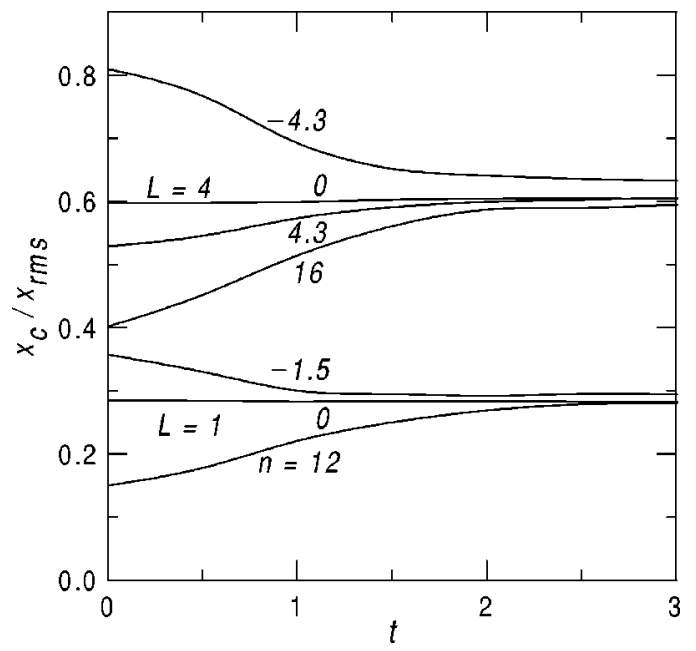

FIG. 3. The ratio $x_{c}(t) / x_{\text {rms }}(t)$ vs time $t$ of expanding vortex states with $L=1, n=-1.5,0,12$ and $L=4, n= \pm 4.3,0,16$.

as large as its value for repulsive condensate. However, there is an interesting difference between the time evolution of this ratio for a fixed $L$ for attractive and repulsive interactions. This ratio decreases with time for attractive interaction and increases with time for repulsive interaction. Hence there is no specific advantage in allowing an attractive vortex to expand for the sake of detection. It will be easier to detect it before expansion, when the vortex core is more pronounced. For the noninteracting case the above ratio remains constant at all times: $\sim 0.28$ for $L=1$ and $\sim 0.60$ for $L=4$. Hence, although the possibility of detection of the repulsive vortex state increases upon free expansion, the opposite is true for attractive interaction. These features of the attractive vortex states upon expansion are the most important results of this investigation clearly displayed in Fig. 3.

We also studied the expansion of vortex states of higher angular momenta in detail. The general features of these states are similar and we present the results for $L=4$ in the following. In Figs. 4(a)-(d) we plot the profile of the complete wave function $|\phi(x, y ; t)|$ vs $x$ and $y$ for $n=-4.3$ for $t=0,1,2$, and 3 . In this attractive case the critical $n$ for collapse is -4.4 [14]. In Figs. 5 and 6 we plot the same for $n=4.3$ and 16, respectively. At all times the wave function is zero on the $y$ axis and is peaked at some finite $x$. Figures 4-6 clearly show the time evolution of the full condensate in these cases. We find from these figures that at a particular time, the vortex-core radius $x_{c}$ is approximately the same in the three cases. However, the radial rms radius $x_{\text {rms }}$ increases as $n$ increases from negative to positive values and consequently, the ratio $x_{c} / x_{\text {rms }}$ increases as we pass from Fig. 4 to Fig. 5 and then to Fig. 6 (from attractive to repulsive). The evolution of the ratio $x_{c} / x_{\text {rms }}$ upon free expansion for $L=4$ and different $n$ is shown in Fig. 3. The results for the $L=4$ case is qualitatively similar to the $L=1$ case and the discussion and comments of the $L=1$ case apply here.

An interesting feature of the freely expanding condensate emerges from the present study. The vortex core radius $x_{c}$ and the radial rms radius $x_{\text {rms }}$ play important roles in this game. The condensed vortex state has the form of a hollow
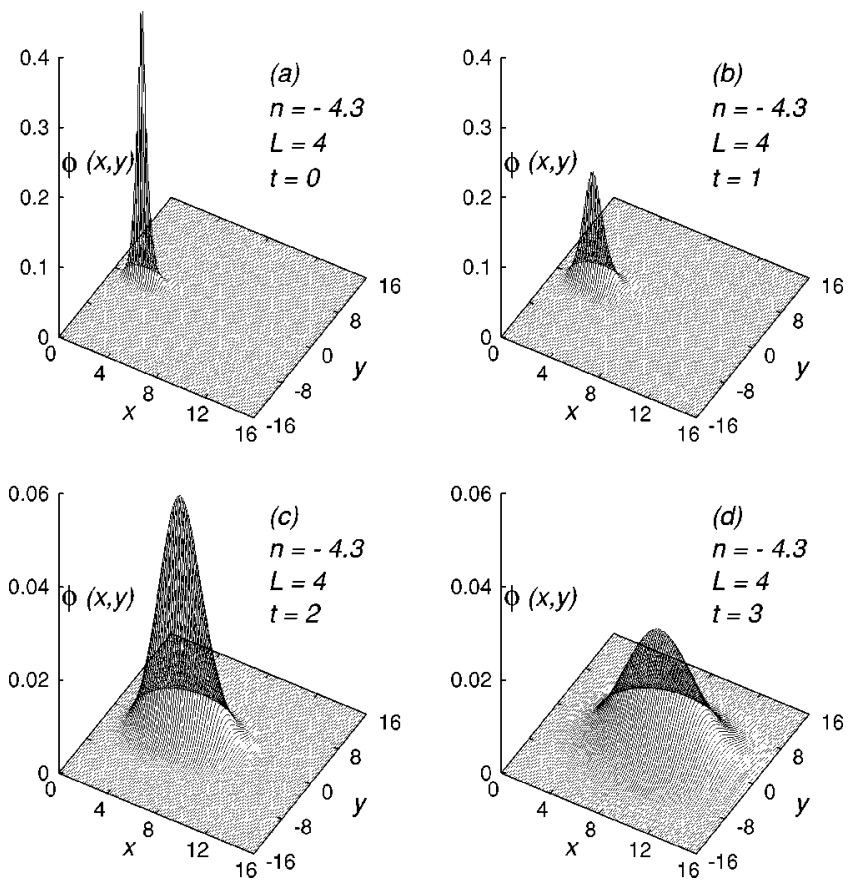

FIG. 4. The wave functions $\phi(x, y)$ of a freely expanding vortex state vs $x$ and $y$ at times $t=0,1,2,3$ for $L=4$ and $n=-4.3$.

cylinder. Qualitatively, $x_{\mathrm{rms}}$ is the radius of the cylinder and $x_{c}$ is the radius of the hollow. The hollow space is more prominent when $x_{c} / x_{\text {rms }}$ is larger. A large value of $x_{c} / x_{\text {rms }}$ facilitates the experimental confirmation of the vortex state. From Figs. 1, 2, 4, 5, and 6 we find that both $x_{c}$ and $x_{\text {rms }}$ increase upon free expansion. However, for an attractive condensate $x_{\text {rms }}$ increases at a rate faster than that of $x_{c}$ so that the above ratio decreases with time. The opposite is true
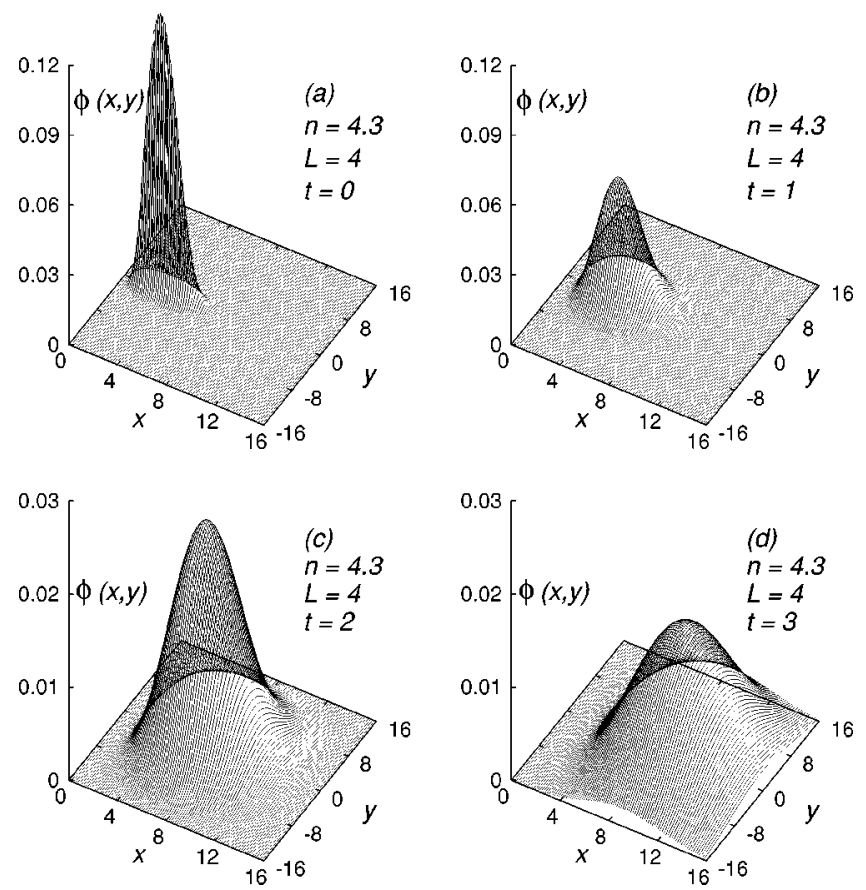

FIG. 5. Same as Fig. 4 for $n=4.3$. 

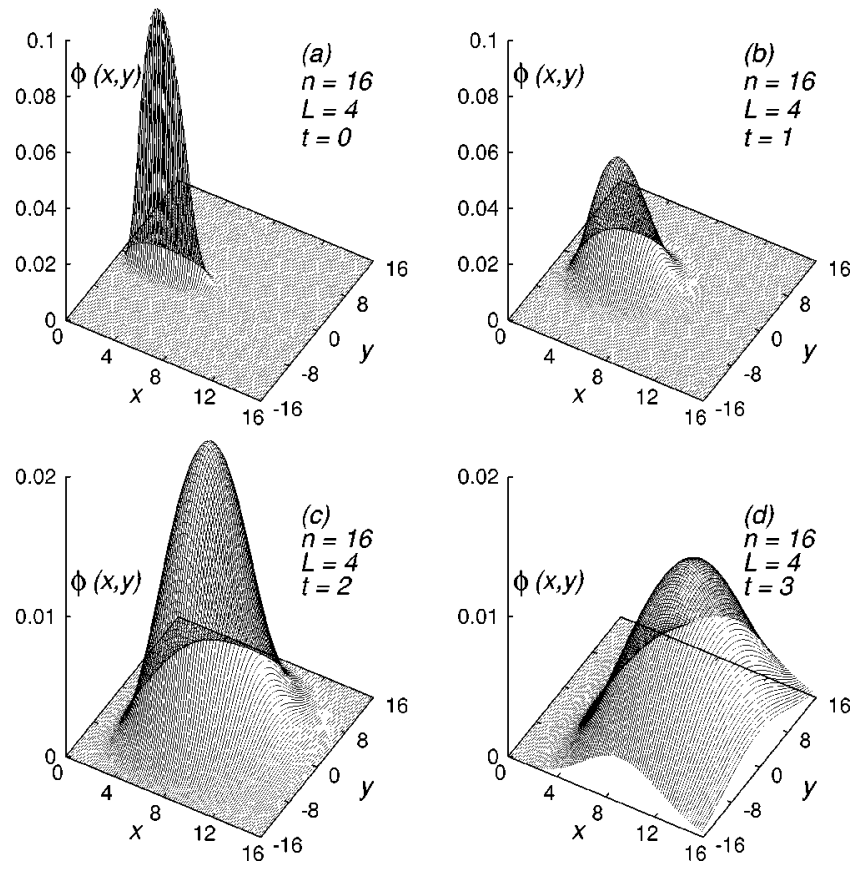

FIG. 6. Same as Fig. 4 for $n=16$.

for a repulsive condensate. For a noninteracting condensate both radii increase at the same rate and the ratio remains constant. The whole scenario is physically understandable. For an attractive condensed vortex state upon free expansion, because of interatomic attraction, the radius of the hollow $x_{c}$ cannot increase fast enough with time compared to the radius of the cylinder. The cylinder as a whole swells at a faster rate than the hollow, making the ratio $x_{c} / x_{\text {rms }}$ decrease with time. The opposite happens for a repulsive condensate, where the atomic repulsion makes the hollow part swell at a much faster rate. For a noninteracting condensate $x_{c}$ and $x_{\mathrm{rms}}$ increase at the same rate leading to a constant $x_{c} / x_{\text {rms }}$. In general for a fixed $L, x_{\text {rms }}$ is much smaller for an attractive condensate, which makes the ratio $x_{c} / x_{\text {rms }}$ much larger than the same for a repulsive condensate.

\section{SUMMARY}

In this paper we present a numerical study of the timedependent Gross-Pitaevskii equation for an axially symmetric trap to obtain insight into the free expansion of vortex states of BEC. The time-dependent GP equation is solved iteratively by discretization using a two-step CrankNicholson scheme [14,17].

The ratio of vortex-core radius to radial rms radius $x_{c} / x_{\text {rms }}(<1)$ is found to play an interesting role in the free expansion of condensed vortex states. The larger this ratio, more prominent is the vortex core and the easier is the possibility of experimental detection of vortex states. For stable vortex states of fixed angular momentum per particle $L$, this ratio for attractive interaction is larger than that for repulsive interaction. Upon free expansion of such states, the ratio for attractive interaction decreases remaining, however, always larger than that for repulsive interaction, which increases after expanion. The ratio increases with $L$ in all cases. This makes the stable attractive vortex states before expansion more suitable for experimental detection.

\section{ACKNOWLEDGMENTS}

This work was supported in part by the Conselho Nacional de Desenvolvimento Científico e Tecnológico and Fundação de Amparo à Pesquisa do Estado de São Paulo of Brazil.
[1] J.R. Ensher, D.S. Jin, M.R. Matthews, C.E. Wieman, and E.A. Cornell, Phys. Rev. Lett. 77, 4984 (1996); K.B. Dadic, M.O. Mewes, M.R. Andrews, N.J. van Druten, D.S. Durfee, D.M. Kurn, and W. Ketterle, ibid. 75, 3969 (1995); D.G. Fried, T.C. Killian, L. Willmann, D. Landhuis, S.C. Moss, D. Kleppner, and T.J. Greytak, ibid. 81, 3811 (1998); F. Pereira Dos Santos, J. Léonard, Junmin Wang, C.J. Barrelet, F. Perales, E. Rasel, C.S. Unnikrishnan, M. Leduc, and C. Cohen-Tannoudji, ibid. 86, 3459 (2001); C.C. Bradley, C.A. Sackett, J.J. Tolett, and R.G. Hulet, ibid. 75, 1687 (1995).

[2] K.W. Madison, F. Chevy, W. Wohlleben, and J. Dalibard, Phys. Rev. Lett. 84, 806 (2000); K.W. Madison, F. Chevy, V. Bretin, and J. Dalibard, ibid. 86, 4443 (2001); M.R. Matthews, B.P. Anderson, P.C. Haljan, D.S. Hall, C.E. Wieman, and E.A. Cornell, ibid. 83, 2498 (1999).

[3] S.K. Adhikari, Phys. Lett. A 281, 265 (2001); 265, 91 (2000); Phys. Rev. A 63, 043611 (2001); J. Phys. B 34, 4231 (2001); A. Gammal, T. Frederico, L. Tomio, and F. Kh. Abdullaev, Phys. Lett. A 267, 305 (2000).

[4] P.A. Ruprecht, M.J. Holland, K. Burnett, and M. Edwards, Phys. Rev. A 51, 4704 (1995); S.K. Adhikari, Phys. Rev. E 63,
056704 (2001); 62, 2937 (2000); M.J. Holland, D.S. Jin, M.L. Chiofalo, and J. Cooper, Phys. Rev. Lett. 78, 3801 (1997); M. Holland and J. Cooper, Phys. Rev. A 53, R1954 (1996); A. Gammal, T. Frederico, and L. Tomio, Phys. Rev. A 64, 055602 (2001).

[5] F. Dalfovo, S. Giorgini, L.P. Pitaevskii, and S. Stringari, Rev. Mod. Phys. 71, 463 (1999).

[6] F. Dalfovo and S. Stringari, Phys. Rev. A 53, 2477 (1996).

[7] F. Dalfovo and M. Modugno, Phys. Rev. A 61, 023605 (2000).

[8] B. Jackson, J.F. McCann, and C.S. Adams, Phys. Rev. A 61, 013604 (1999).

[9] D.L. Feder, C.W. Clark, and B.I. Schneider, Phys. Rev. Lett. 82, 4956 (1999).

[10] J.J. García-Ripoll and V.M. Pérez-García Phys. Rev. Lett. 84, 4264 (2000); V.M. Pérez-García and J.J. García-Ripoll, Phys. Rev. A 62, 033601 (2000); G.M. Kavoulakis, B. Mottelson, and C.J. Pethick, ibid. 62, 063605 (2000).

[11] R.J. Marshall, G.H.C. New, K. Burnett, and S. Choi, Phys. Rev. A 59, 2085 (1999).

[12] L. Dobrek, M. Gajda, M. Lewenstein, K. Sengstock, G. Birkl, and W. Ertmer, Phys. Rev. A 60, R3381 (1999). 
[13] A.A. Svidzinsky and A.L. Fetter, Phys. Rev. A 62, 063617 (2000).

[14] S. K. Adhikari, Phys. Rev. E 65, 016703 (2002).

[15] J.L. Roberts, N.R. Claussen, S.L. Cornish, E.A. Donley, E.A. Cornell, and C.E. Wieman, Phys. Rev. Lett. 86, 4211 (2001).

[16] E.P. Gross, Nuovo Cimento 20, 454 (1961); L.P. Pitaevskii,
Zh. Éksp. Teor. Fiz. 40, 646 (1961) [Sov. Phys. JETP 13, 451 (1961)].

[17] S.E. Koonin and D.C. Meredith, Computational Physics Fortran Version (Addison-Wesley, Reading, MA, 1990), pp. 169180. 